\title{
Familial Cholestatic Syndromes
}

\author{
Richard Thompson \\ Paediatric Hepatology, King's College London School of Medicine, London, UK
}

\begin{abstract}
Key Words
Familial cholestatic syndromes • Alagille syndrome • Arthrogryposis, renal dysfunction and cholestasis syndrome $\cdot$ Familial intrahepatic cholestasis 1 deficiency • Bile salt export pump deficiency $\cdot$ Multidrug resistance protein 3 deficiency
\end{abstract}

\begin{abstract}
The term 'familial' is an unfortunate one. Although the definition includes 'hereditary', the inference is that it will usually have occurred in other family members. For many of the conditions described in this article this is often not the case, and for this reason, 'familial' conditions may be overlooked when reaching a diagnosis. Cholestasis is defined differently by clinicians, pathologists and biochemists. In addition, what is meant by cholestatic liver disease very often varies for paediatricians and adult hepatologists. However, the pathogenic mechanisms are increasingly similar. Biliary atresia, which is the most frequent cholestatic liver disease presenting to paediatricians, is strikingly different from most diseases as there appears to be no 'late-onset' equivalent and it is almost never familial. Several international collaborations have now been established in the hope of overcoming these hurdles (http://www.barcnetwork.org/, http://www.orpha. net/nestasso/EFBAR/, http://www.biliary-atresia.com/index frameset_ebar.html). On a day-to-day basis, paediatric hepatologists are faced with a group of infants with jaundice in whom the cause is not readily apparent. In many ways, this
\end{abstract}

has been worsened by the fact that we have known for a long time that a significant proportion will improve spontaneously, whereas others will do badly. In the first few months of life, we still have difficulty forecasting outcomes for individual patients. The underlying cause, at least in the group with a poor outcome, is now becoming apparent in an increasing number of cases. Most of the improvements in our unravelling of this group of children have come about as the result of advances in our understanding of the genetic aetiology. It has also become clear that genetic cholestatic liver disease is not restricted to paediatrics. The exact extent to which genetics contributes to 'late-onset' disease is only now being unravelled.

Copyright $\odot 2008$ Nestec Ltd., Vevey/S. Karger AG, Basel

Genetics is not yet the first port of call in making a diagnosis in a cholestatic infant, though it may be soon [4]. The fact that cholestatic diseases are now being classified on the basis of their genetic cause makes it easier to identify phenotypic variations. These phenotypic differences improve both diagnosis and prognosis. They help us decide on the condition of a child, or at least suggest which gene or protein should be investigated. The phenotype extends to prognosis and particularly treatment outcomes, and a better understanding of these factors helps the clinician and the family alike. The major familial cholestatic syndromes are summarized in table 1.

\begin{tabular}{|c|c|}
\hline KARGER & $\begin{array}{l}\text { (C) } 2008 \text { Nestec Ltd., Vevey/S. Karger AG, Basel } \\
0517-8606 / 08 / 0663-0121 \$ 24.50 / 0\end{array}$ \\
\hline $\begin{array}{l}\text { Fax +41 } 613061234 \\
\text { E-Mail karger@karger.ch } \\
\text { www.karger.com }\end{array}$ & $\begin{array}{l}\text { Accessible online at: } \\
\text { www.karger.com/ane }\end{array}$ \\
\hline
\end{tabular}

Richard Thompson

Institute of Liver Studies

King's College Hospital

Denmark Hill, London SE5 9RS (UK)

E-Mail richard.j.thompson@kcl.ac.uk 
Table 1. The major familial cholestatic syndromes

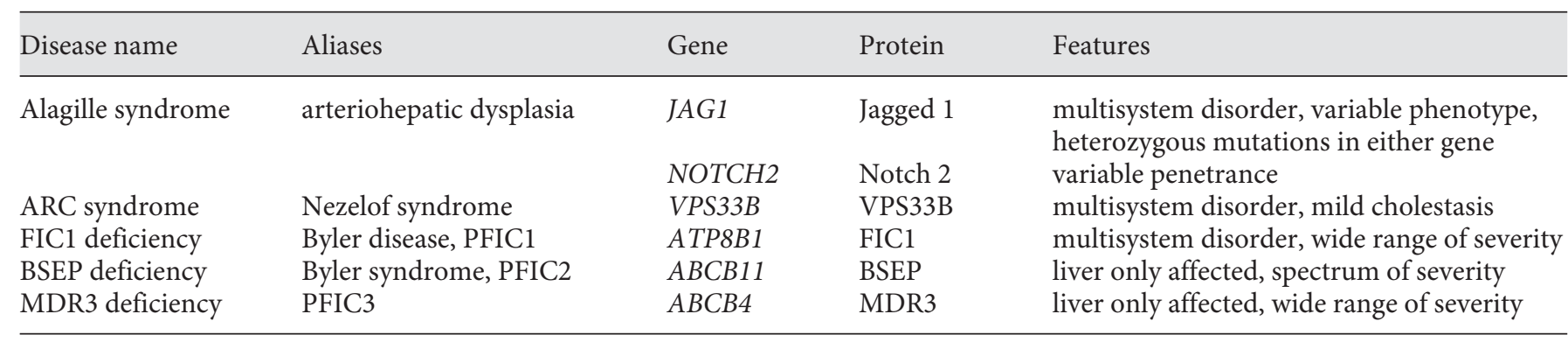

\section{Alagille Syndrome}

Alagille syndrome has always been a clinical diagnosis. In most cases, genetics would not be needed to diagnose an individual patient. Mutations were first identified in JAGGED1, which encodes a ligand in the Notch signalling pathway $[5,6]$. Subsequently, in other patients, mutations have been identified in the gene encoding one of the Notch receptors, NOTCH2 [7]. All in all, mutations have only been found on a single allele of the gene in question. One presumes that a loss of both alleles would be lethal, and certainly the mouse data would support this [8]. It is now clear that a genetic diagnosis can be arrived at in the vast majority of such individuals [9]. In many ways, this has only complicated things, particularly, as all published data concerning the frequency of different features and, more importantly, outcome data relate to patients diagnosed clinically. Although most patients have an identifiable genetic 'cause', it has long been known that family members can have similar, though milder, features. It is now clear that these relatives often share the same genetic abnormality. By no stretch of imagination could these individuals be said to have Alagille syndrome. This problem is all the greater when realising that in the general population, we have no idea of the frequency of mutations in the genes involved. Therefore, the diagnosis should probably remain a clinical one, until the true penetrance of mutations is established and the mechanisms that determine the phenotype are understood.

\section{Arthrogryposis, Renal Dysfunction and Cholestasis Syndrome}

Luckily, arthrogryposis, renal dysfunction and cholestasis syndrome (ARC) is rare. However, it does represent an important disease mechanism. In the same way as Alagille syndrome, it was described clinically. The phenotype as described was very severe, particularly in terms of the arthrogryposis, even though the liver disease was relatively mild. The gene mutated in the majority of cases has been identified as VPS33B [10]. Along with all the conditions described in the rest of this article, the mutations are recessive. It has long been known that many of the functions of the hepatocyte are highly polarised, with very different functions taking place at the basolateral and canalicular membranes (fig. 1). The protein encoded by VPS33B appears to be implicated in correctly sorting some proteins to the canalicular domain, and is not itself a trans-membrane transporter. It seems highly likely that other (possibly similar) phenotypes will be manifest if other proteins in this pathway are defective. However, as for the Alagille syndrome, patients have already been identified who do not have the complete phenotype [11]. This is important, as such patients would not have been classified as ARC previously, as they did not meet the clinical criteria. Again, this raises the, as yet unanswerable, question of how many patients with less severe phenotypes harbour mutations in these genes.

\section{Familial Intrahepatic Cholestasis 1 Deficiency}

Familial intrahepatic cholestasis 1, or FIC1, is the name generally applied to the protein encoded by ATP8B1 [12]. This prosaic genetic nomenclature indicates that the protein is predicted to be P-type ATPase and is thus a relative of the Wilson disease gene. However, as discussed later, its function is probably quite different. FIC1 deficiency has been described as causing both benign recurrent intrahepatic cholestasis (BRIC) and progressive familial intrahepatic cholestasis (PFIC). In truth, it is evident that there are patients that fall between these 2 defined diseases, and that FIC1 deficiency 
is really a spectrum ranging from mild to severe liver disease [13]. This is clinically important as liver disease may progress, even in patients initially diagnosed with BRIC, often on the basis of a late presentation. Therefore, the term 'BRIC' is somewhat misleading and, indeed, may be falsely reassuring to both patients and their carers.

Many P-type ATPases are ion transporters, as is the case with the Wilson disease-associated protein. FIC1 on the other hand is thought to be one of several aminophospholipid flippases. The requirement for the maintenance of lipid asymmetry in biological membranes is clear, such that we probably have several of these proteins, expressed in a variety of tissues. The correct distribution of lipids between bilayers may well be essential for the sorting of proteins to the correct compartment. Equally, it is likely that the correct lipid composition of the plasma membrane is essential for the normal function of other protein therein. FIC1 deficiency not only manifests as cholestatic liver disease, but also with a highly variable set of other features. The most common are diarrhoea, hearing loss, renal tubular dysfunction and growth failure. Somewhat surprisingly, individuals can have severe manifestation of one aspect of the disease, with minimal features in another system. The multisystem nature of the disease is supported by the widespread expression of the ATP $8 B 1$ gene [12]. In the liver, several proteins are not present, or present in significantly reduced quantities, in the canalicular membrane of patients with FIC1 deficiency. This is consistent with the hypothesis that abnormal membrane lipids are leading to abnormality of protein sorting similar in nature to ARC syndrome. Still, none of the proteins missing are known to be critical to bile formation or associated with cholestasis. In particular, immunohistochemical staining for the bile salt export pump (BSEP, itself discussed in more detail below) appears normal in FIC1 deficiency; however, recent data support another hypothesis [14]. Although largely based on a mouse model, this work does suggest that the FIC1 protein facilitates the movement of aminophospholipids from the outer to the inner leaflets of the plasma membrane [14], as was believed. The normal canalicular membrane needs to maintain a high degree of detergent resistance in order to prevent damage from the detergent effect of bile itself. The presence of excess aminophospholipids in the outer leaflet would reduce this detergent resistance. Using a short-term ex vivo bile acid infusion experiment, these investigators found evidence that the availability of aminophospholipids for extraction by bile acids is greatly increased in the knockout model. Furthermore, some of the canalicular ectoenzymes in the knockout livers are eluted

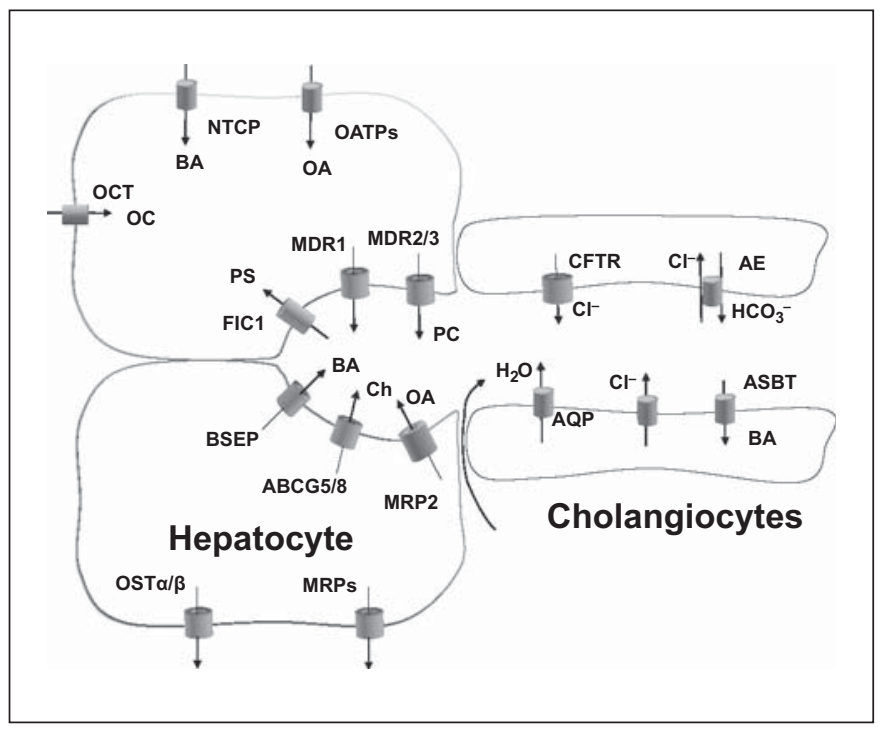

Fig. 1. The major transporters involved in bile formation in the hepatocyte and cholangiocyte membranes, many of which are now associated with familial cholestasis. ABCG5/8 = Heterodimeric cholesterol transporter; $\mathrm{AE}=$ anion exchanger; $\mathrm{ASBT}=$ apical sodium-dependent bile acid transporter; $\mathrm{AQP}=$ aquaporin; $\mathrm{BA}=$ bile acids; CFTR = cystic fibrosis transmembrane conduc tance regulator; $\mathrm{Ch}=$ cholesterol; FIC1 $=$ familial intrahepatic cholestasis protein $1 ; \mathrm{MDR}=$ multidrug resistance protein; $\mathrm{MRP}=$ multidrug resistance-associated protein; $\mathrm{NTCP}=$ sodi$\mathrm{um}$ /taurocholate cotransporting polypeptide; $\mathrm{OA}=$ organic anions; OATP = organic anion-transporting poplypetide; $\mathrm{OC}=$ organic cations; $\mathrm{OCT}=$ organic cation transporter; OST $=$ heterodimeric organic solute transporter; PS = phosphatidylserine.

in a bile salt-dependent fashion. These are some of the same enzymes previously shown to be absent from the human liver. This raises the possibility that these proteins have been removed from the canalicular membrane of human disease liver, instead of being subjected to a trafficking or targeting defect. This has not yet been proven in humans and it still does not account for cholestasis, as no transporter critical to bile formation has been shown to be absent. For these large proteins, it seems most likely that it is their function that is affected by the abnormal lipid composition of the canalicular membrane. The same may be the case in the other affected organs. In the ear, the kidney or the pancreas, it is clearly not the action of bile acids removing proteins that leads to the phenotype; there seems little doubt that the correct lipid composition of the membrane is critical to many functions. We are left with the additional complication of the highly variable phenotype. We know that there are several other aminophospholipid transporters in the human 
proteome. It seems likely that in FIC1 deficiency, the disease is only manifest where other proteins are not adequately compensating for the loss of FIC1 function. The extrahepatic manifestations of FIC1 deficiency limit the utility of liver transplantation. In particular, diarrhoea can get worse, and growth failure may not be impacted at all. The debilitating pruritus may be enough to justify the undertaking, but it cannot be regarded as a cure.

FIC1 deficiency was once called 'Byler disease', after the Amish family in which it was first described. Byler bile is the description of the canalicular contents seen in FIC1 deficiency on transmission electron microscopy. These unusual aggregates are not quite diagnostic of this condition, but are certainly highly suggestive. The knockout mouse develops a morphology indicative of Byler bile when fed cholic acid [14]. In the mouse, the appearances are highly suggestive of the granular components being formed from damaged microvilli, derived from the canalicular membrane. This loss of membrane surface area may, in itself, contribute to cholestasis. If the main mechanism by which the lack of FIC1 exerts its effect on the liver is by rendering the canalicular membrane susceptible to bile salt damage, it is surprising that the degree of morphological disruption seen on light microscopy is not greater. Yet again, it seems likely that even here, in the liver, the impact of the loss of FIC1 function is reduced by the presence of other lipid transporters and that a complete loss of aminophospholipid internalisation would probably lead to a significantly more severe liver disease.

\section{BSEP Deficiency}

The major bile salt transporter of the canalicular membrane, now referred to as the BSEP, is encoded by the gene $A B C B 11$. In a fashion similar to that seen in FIC1 deficiency, patients have been identified with both mild (BRIC) [15] and severe (PFIC) [16, 17] phenotypes. The nomenclature is again unfortunate as there are undoubtedly patients all along the spectrum in between the $2 \mathrm{ex}-$ tremes. This is clinically extremely important as several so-called BRIC patients have features that suggest progressive liver disease, and the fact that a disease presents late, and may appear to remit, should not be taken as evidence that it will not cause later problems. The pathophysiology mechanisms leading to BSEP deficiency are somewhat easier to understand. A complete, or partial, failure of bile acid transport, at the canalicular membrane, is going to have an obvious effect on hepatocellular function. It is clear that the hepatocyte disarray is much greater than that seen in FICl deficiency, often showing considerable giant cell transformation in infancy. BSEP appears not to be expressed outside the liver, and therefore, the clinical feature can entirely be accounted for by a failure of bile salt transport at the canalicular membrane. The clinical manifestations are those of severe cholestasis, though, as bilirubin is transported quite separately from bile salts, the degree of jaundice can markedly underestimate the degree of cholestasis. Liver transplantation is an excellent form of treatment for BSEP deficiency.

In children, hepatocellular carcinoma (HCC) is extremely rare. Cholangiocarcinoma is even rarer. On the other hand, hepatoblastoma is the commonest hepatocyte-derived malignancy, along with the much rarer fibrolamellar HCC. Both of these malignancies occur against the background of a normal liver, not in the context of chronic liver disease. In children, HCC is seen with some specific diseases, notably, classical tyrosinaemia and, to a lesser extent, glycogen storage diseases. Ten young children with HCC and BSEP deficiency were reported following a search for children with HCC against the background of a severe neonatal hepatitis picture [18]. The exact risk for each patient is difficult to assess, particularly as many patients are transplanted before developing this complication. In a subsequent study, 2 children with cholangiocarcinomata, again presenting against the background of a neonatal hepatitis, were found to have BSEP deficiency [19]. A subsequent analysis of 128 children with mutation-proven, severe BSEP deficiency found that 19 had had the one or the other malignancy [17]. The risk appears to be much greater if the individual has 2 protein-truncating mutations ( 38 vs. 10\% in other genotypes). Many patients had had early transplantation, without developing malignancy. In the absence of transplantation, the risk must be considerably higher. All these patients had clinically severe disease; there was no apparent correlation between disease severity and risk of malignancy. Considering that other children with persistent cholestasis do not seem to develop malignancy, one is forced to question the mechanism by which the neoplastic change occurs. The obvious candidate of damage induced by the retained bile salts is thrown into doubt by several of the above observations. It is only possible to speculate as to the true mechanism. However, it is certainly possible that BSEP is normally transporting low levels of something other than bile salts, which is caused to accumulate in its absence, or perhaps, BSEP has some other function entirely, the complete absence of which 
leads to malignancy. Whatever the mechanism, the risk is real and must be taken into account when considering all modes of treatment, other than transplantation, be they pharmacological, surgical or genetic. All will presumably leave a risk of malignancy, requiring very close monitoring.

\section{Multidrug Resistance Protein 3 Deficiency}

In the course of human evolution, we have become extremely efficient at extracting fat from our diet. In much of the world, where calories are in abundance, we are paying the price. However, in order to be so good at extracting fat from our diet we have evolved the ability to synthesise highly detergent bile acids. Bile acids, by their very nature, are toxic. When retained in the liver, they lead to severe disease; as seen in BSEP deficiency. However, after transportation into bile they achieve millimolar concentrations. Therefore, bile itself has the potential to cause severe damage to the biliary tree. Indeed, whatever triggers sclerosing cholangitis, or biliary atresia, bile in the form of bile acids does much of the damage. Humans and many mammals have had to evolve mechanisms to minimise the toxic effects of bile acids. A major mechanism by which we do this is by enabling the formation of mixed micelles. These are composed of bile acids and other lipids, most notably phosphatidylcholine (PC). The entry of lipids, particularly PC, into bile to form micelles is closely coupled to bile acid flux. The data relating to FIC1 deficiency, discussed above, suggest that bile acids can extract lipids from the canalicular membrane in a rather non-specific fashion if they are made available. Normally, this does not happen, as most of the membrane is detergent resistant. Instead, PC seems to be extracted in a highly specific fashion. We now recognise multidrug resistance protein 3 (MDR3) as being essential for the entry of PC into bile [20]. The availability of PC in the outer leaflet of the canalicular membrane appears to be tightly controlled by MDR3. Therefore, even a minor loss of MDR3 function would be expected to change the composition of bile. Indeed, a whole range of phenotypes have been described associated with reduced MDR3 function, including propensity of gallstone formation [21, 22]. Patients with only one defective copy can manifest symptoms under certain circumstances, for example during pregnancy $[23,24]$, though they are otherwise well. The severe end of the spectrum, PFIC, seems relatively rare. This condition is readily distinguished from FIC1 and BSEP deficiencies as it is associated with elevated levels of circulating $\gamma$-glutamyl transferase. However, as MDR3 mutations are increasingly sought, late-onset MDR3-related disease, including small duct sclerosing cholangitis, AMA-negative primary biliary cirrhosis and even cryptogenic cirrhosis are being identified. Whether MDR3 really plays a major role in cholesterol cholelithiasis remains to be seen. However, in pedigrees with multiple affected individuals, particularly if the stones are intrahepatic, mutations within this gene are not uncommon [25].

\section{Conclusions}

In the last 10 years, our understanding of the molecular mechanisms involved in bile formation and cholestasis has improved dramatically. Analysis of families segregating different forms of liver disease has played a significant part in these advances. In addition, we can now see that some late-onset conditions have, in some cases, a genetic cause, and in others, a genetic susceptibility. The full extent to which late-onset liver diseases are actually milder versions of these conditions remains to be seen; we have probably only scratched the surface so far. Within paediatrics, it is now essential to convert this understanding into better outcomes for patients. In the United States, a multi-centre collaboration is seeking to do just that [26]. Much of the published retrospective data include patients we would now consider to be poorly characterised. With our greatly improved techniques and knowledge, we should now be able to make diagnoses more quickly, understand the disease processes better and plan treatment and trials in light of this knowledge.

References

1 Biliary Atresia Research Consortium. http:// www.barcnetwork.org/.

2 European Federation of Biliary Atresia Research. http://www.orpha.net/nestasso/ EFBAR/.

3 European Biliary Atresia Registry. http:// www.biliary-atresia.com/index_frameset_ ebar.html.

4 Liu C, Aronow BJ, Jegga AG, et al: Novel resequencing chip customized to diagnose mutations in patients with inherited syndromes of intrahepatic cholestasis. Gastroenterology 2007;132:119-126.

5 Oda T, Elkahloun AG, Pike BL, et al: Mutations in the human Jagged1 gene are responsible for Alagille syndrome. Nat Genet 1997; 16:235-242. 
-6 Li L, Krantz ID, Deng Y, et al: Alagille syndrome is caused by mutations in human Jagged1, which encodes a ligand for Notch1. Nat Genet 1997;16:243-251.

7 McDaniell R, Warthen DM, Sanchez-Lara $\mathrm{PA}$, et al: NOTCH2 mutations cause Alagille syndrome, a heterogeneous disorder of the notch signaling pathway. Am J Hum Genet 2006;79:169-173.

$>8$ Xue Y, Gao X, Lindsell CE, et al: Embryonic lethality and vascular defects in mice lacking the Notch ligand Jagged1. Hum Mol Genet 1999;8:723-730.

$>9$ Warthen DM, Moore EC, Kamath BM, et al: Jagged1 (JAG1) mutations in Alagille syndrome: increasing the mutation detection rate. Hum Mutat 2006;27:436-443.

10 Gissen P, Johnson CA, Morgan NV, et al: Mutations in VPS33B, encoding a regulator of SNARE-dependent membrane fusion, cause arthrogryposis-renal dysfunctioncholestasis (ARC) syndrome. Nat Genet 2004;36:400-404.

- 11 Bull LN, Mahmoodi V, Baker AJ, et al: VPS33B mutation with ichthyosis, cholestasis, and renal dysfunction but without arthrogryposis: incomplete ARC syndrome phenotype. J Pediatr 2006;148:269-271.

12 Bull LN, van Eijk MJ, Pawlikowska L, et al: A gene encoding a P-type ATPase mutated in two forms of hereditary cholestasis. Nat Genet 1998;18:219-224.
13 van Mil SW, Klomp LW, Bull LN, et al: FIC1 disease: a spectrum of intrahepatic cholestatic disorders. Semin Liver Dis 2001;21:535544.

4 Paulusma CC, Groen A, Kunne C, et al: Atp8b1 deficiency in mice reduces resistance of the canalicular membrane to hydrophobic bile salts and impairs bile salt transport. Hepatology 2006;44:195-204.

15 van Mil SW, van der Woerd WL, van der Brugge $G$, et al: Benign recurrent intrahepatic cholestasis type 2 is caused by mutations in ABCB11. Gastroenterology 2004;127:379384.

16 Strautnieks SS, Bull LN, Knisely AS, et al: A gene encoding a liver-specific ABC transporter is mutated in progressive familial intrahepatic cholestasis. Nat Genet 1998;20: 233-238.

17 Strautnieks SS, Byrne JA, Pawlikowska L, et al: Severe bile salt export pump deficiency: 82 different ABCB11 mutations in 109 families. Gastroenterology 2008;134:1203-1214.

18 Knisely AS, Strautnieks SS, Meier Y, et al: Hepatocellular carcinoma in ten children under five years old with bile salt export pump deficiency. Hepatology 2006;44:478486.

19 Scheimann AO, Strautnieks SS, Knisely AS, et al: Mutations in bile salt export pump (ABCB11) in two children with progressive familial intrahepatic cholestasis and cholangiocarcinoma. J Pediatr 2007;150:556-559.
20 de Vree JM, Jacquemin E, Sturm E, et al: Mutations in the MDR3 gene cause progressive familial intrahepatic cholestasis. Proc Natl Acad Sci USA 1998;95:282-287

21 Rosmorduc O, Hermelin B, Poupon R: MDR3 gene defect in adults with symptomatic intrahepatic and gallbladder cholesterol cholelithiasis. Gastroenterology 2001;120: 1459-1467.

22 Jacquemin E, De Vree JM, Cresteil D, et al: The wide spectrum of multidrug resistance 3 deficiency: from neonatal cholestasis to cirrhosis of adulthood. Gastroenterology 2001;120:1448-1458.

23 Dixon PH, Weerasekera N, Linton KJ, et al: Heterozygous MDR3 missense mutation associated with intrahepatic cholestasis of pregnancy: evidence for a defect in protein trafficking. Hum Mol Genet 2000;9:12091217.

24 Jacquemin E, Cresteil D, Manouvrier S, et al: Heterozygous non-sense mutation of the MDR3 gene in familial intrahepatic cholestasis of pregnancy. Lancet 1999;353:210 211.

25 Rosmorduc O, Poupon R: Low phospholipid associated cholelithiasis: association with mutation in the MDR3/ABCB4 gene. Orphanet J Rare Dis 2007;2:29.

26 Cholestatic Liver Disease Consortium. http://rarediseasesnetwork.epi.usf.edu/clic/ index.htm. 